\section{Contractilidad ventricular. Fisiología y proyección clínica}

\author{
RAÚL J. DOMENECH ${ }^{1}$, VÍCTOR M. PARRA ${ }^{1,2}$
}

\section{Ventricular contractility. Physiology and clinical projection}

The contractile state of the heart is the result of myocardial contractility, the intrinsic mechanism that regulates the force and the shortening of the ventricle and determines the ventricular ejection volume. However, the ejection volume is also modulated by ventricular preload (diastolic ventricular volume) and afterload (resistance to ejection). Accordingly, a decrease in contractility may be masked by changes in preload or afterload, maintaining a normal ejection volume and delaying the diagnosis of myocardial damage. Thus, it is necessary to develop a non-invasive method to measure contractility in the clinical practice. We review in this article the basic principles of cardiac contraction, the concept of contractility and its measurement with the ventricular pressure-volume loop, an experimental method that also measures most of the hemodynamic variables of the cardiac cycle including preload, afterload, ventricular work, ventricular lusitropy and arterial elastance. This method has been recently validated in cardiac patients and allows to evaluate the evolution of contractility in heart failure in a non invasive way. Although some modifications are still necessary, it will probably have an extensive use in practical cardiology in the near future.

(Rev Med Chile 2016; 144: 771-779)

Key words: Cardiovascular Physiological Phenomena; Cardiovascular System; Myocardial Contraction; Physiology.

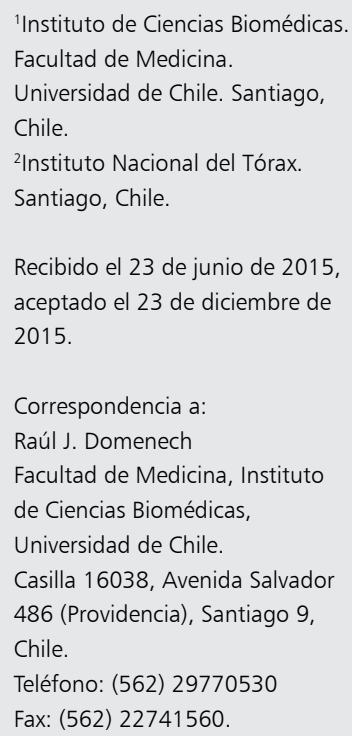

L a pregunta no es justa porque ustedes tampoco saben la respuesta (respuesta de un estudiante de medicina durante un examen cuando se le preguntó ¿qué es "contractilidad”?).

\section{Fisiología y definiciones}

La función sistólica del ventrículo izquierdo determina la eyección de sangre hacia la aorta. El factor central en esta eyección es la capacidad contráctil del sarcómero miocárdico ${ }^{1}$ para desarrollar fuerza y acortarse gracias a la formación de puentes entre los filamentos de actina y miosina y el deslizamiento de estos filamentos entre sí (Figura 1). El número de puentes y su velocidad de recambio depende de:
1. Proceso intrínseco del sarcómero. Se inicia con la entrada de una pequeña cantidad de ión calcio a la célula por los canales $\mathrm{L}$ de calcio durante la despolarización celular, lo que a su vez gatilla la liberación desde el retículo sarcoplásmico hacia el citosol de una gran cantidad de calcio, el que al unirse a troponina en el miofilamento delgado activa la formación de puentes y su recambio. El estado contráctil determinado por este proceso intrínseco se denomina contractilidad, y es regulado por la actividad $\beta$ adrenérgica y diversas substancias con acción inótropa.

2. Acciones externas al sarcómero, principalmente:

a) Precarga ventricular. Es la longitud del sarcómero al inicio de la contracción. Al dilatarse el ventrículo mayor es la distensión del sarcómero, lo 


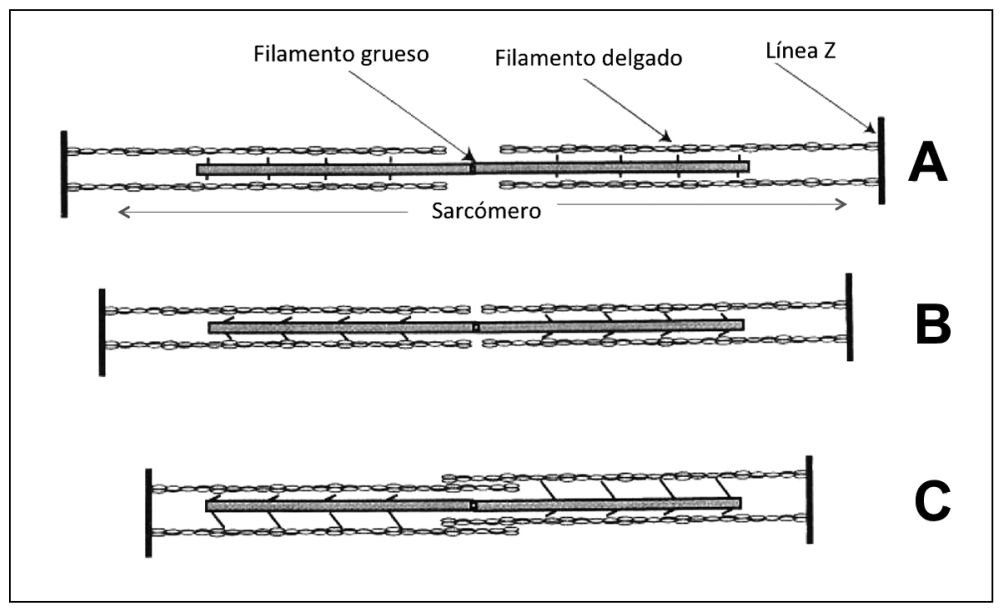

Figura 1.

que disminuiría la distancia entre los miofilamentos aumentando la sensibilidad de ellos al calcio, lo cual significa mayor número de puentes y mayor velocidad de recambio, con aumento del volumen expulsivo y gasto cardiaco ${ }^{1}$. Esto equivale a la ley de Starling. La precarga se evalúa por el volumen ventricular de fin de diástole.

b) Postcarga ventricular. Es la dificultad del ventrículo a la eyección de la sangre, estando aumentada en casos como estenosis aórtica, hipertensión arterial, y al aumentar la rigidez de la aorta. La postcarga también aumenta en el ventrículo dilatado, debido a que el estrés parietal aumenta al incrementar el volumen ventricular según la ley de Laplace ${ }^{1}$, reflejando una mayor resistencia a expulsar la sangre aún cuando no exista un obstáculo mecánico en la salida ventricular. Con el aumento de la postcarga, mayor es el número de puentes enganchados desarrollando fuerza y menor el número de puentes en reciclaje, por lo cual menor es la velocidad y la magnitud del acortamiento y por ende el volumen expulsivo y gasto cardiaco. La disminución de la postcarga en cambio produce el fenómeno opuesto, con aumento del volumen expulsivo y gasto cardiaco. La postcarga se evalúa por la presión ventricular durante la eyección y en la práctica por la magnitud de la presión arterial sistólica.

Nótese que tanto el proceso intrínseco del sarcómero como los efectos de cambios de precarga y postcarga dependen del acceso de calcio a los miofilamentos y la mecánica de los puentes. La acción de precarga y postcarga es realmente una modulación del proceso intrínseco, lo que hace elusiva su separación. Sin embargo, en clínica es útil distinguir un cambio del estado contráctil causado por una modificación de la contractilidad, del causado por una modificación de precarga o postcarga. Los cambios de precarga y postcarga ocurren permanentemente en diversas condiciones fisiológicas y patológicas, pudiendo enmascarar una disminución de la contractilidad, por ejemplo en isquemia miocárdica o sobrecarga ventricular crónica (valvulopatía aórtica o mitral), manteniendo un volumen expulsivo normal por un aumento de precarga y/o una disminución de postcarga, demorando el diagnóstico del daño miocárdico y empeorando su pronóstico. La medición de la contractilidad permitiría tomar decisiones terapéuticas en forma precoz. Desgraciadamente, ninguno de los índices de contractilidad invasivos $(\mathrm{dp} / \mathrm{dt})$ o subrogantes no invasivos (fracción de eyección, índices de deformación ventricular como strain y strain rate) usados actualmente en clínica son independientes de la precarga y postcarga ventricular ${ }^{1,2}$, si bien la deformación ventricular, más específicamente strain rate, guarda una buena correlación con ella y tendría valor pronóstico ${ }^{3-7}$.

\section{Medición de la contractilidad}

\section{Medición de la contractilidad "in vitro"}

Para conocer en un estudio in vitro si una droga modifica el estado contráctil del miocardio independiente de precarga y postcarga, se mide la 
fuerza isométrica (postcarga cero porque no hay acortamiento) desarrollada por un músculo papilar a partir de una determinada longitud inicial (precarga), o bien la magnitud de su acortamiento para un determinado peso a levantar (postcarga) y una determinada longitud inicial (precarga). La comparación de los resultados en la condición basal y bajo la acción de la droga demostrará si se modificó la contractilidad.

En cambio, descartar el efecto de la precarga y postcarga para conocer la contractilidad en el corazón entero en el animal de experimentación es difícil, y más aún en el hombre en forma no invasiva. Sólo recientemente, gracias al avance del conocimiento de la función ventricular y el desarrollo de técnicas no invasivas para medir volúmenes ventriculares se ha logrado una buena aproximación a la medición de contractilidad de la cámara ventricular in vivo, extendiendo su aplicación al ser humano. A continuación se describen las bases experimentales de esta aproximación.

\section{Medición de la contractilidad "in vivo" en el animal de experimentación. Elastancia y "bucle ventricular"}

La estimación más aceptada actualmente de la contractilidad ventricular es en base a las curvas que relacionan la presión ventricular con el volumen ventricular durante la contracción del miocardio ${ }^{8-10}$. Midiendo la presión y el volumen ventricular desarrollados en el tiempo a partir de diferentes volúmenes ventriculares de fin de diástole (diferentes precargas) se puede construir curvas de la relación presión-volumen instantáneas para determinados momentos de la sístole (isócronas). La Figura 2 muestra esta relación en el corazón del perro ${ }^{10}$. Se muestran 5 curvas cada $45 \mathrm{~ms}$ entre los 40 y $220 \mathrm{~ms}$ después de iniciada la sístole. Cada curva en línea continua (prácticamente una recta en análisis de regresión) corresponde a la presión desarrollada por el ventrículo durante su contracción, ya sea isovolúmica o auxobárica (con acortamiento), para diferentes volúmenes ventriculares en un momento determinado después de iniciada la contracción. Las proyecciones de las curvas coinciden en la abscisa en el volumen denominado $V_{0}$ (volumen ventricular a presión ventricular cero) con muy escasa dispersión. La pendiente (cambio de presión por unidad de cambio de volumen) de cada curva se denomina elastancia, E, y se expresa:

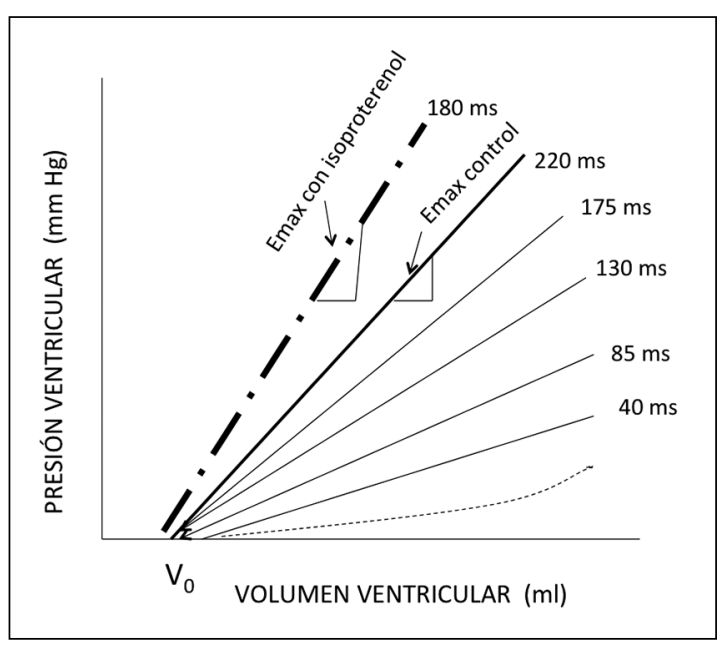

Figura 2.

$$
\mathrm{E}(\mathrm{t})=\mathrm{P}(\mathrm{t}) /\left[\mathrm{V}(\mathrm{t})-\mathrm{V}_{0}\right]
$$

en que $\mathrm{P}(\mathrm{t})$ y $\mathrm{V}(\mathrm{t})$ equivalen a la presión y al volumen ventricular medidos en el momento t. La elastancia corresponde a la rigidez alcanzada por la pared ventricular y denota el estado contráctil del miocardio en ese momento. Nótese que E (pendiente de la curva) va aumentando en el tiempo hasta llegar a un valor de elastancia máxima (Emax), a los $220 \mathrm{~ms}$ de iniciada la contracción (línea continua en negrita en Figura 2), cuando el ventrículo desarrolla el máximo de presión al final de la eyección. Emax no puede ser alterada por cambios de la precarga puesto que se construye con diferentes volúmenes ventriculares de fin de diástole, es decir con diferentes precargas. Así mismo, Emax no puede ser alterada por cambios de la postcarga porque su curva representa la máxima presión de fin de sístole que el ventrículo puede desarrollar para cada volumen ventricular. Es decir, Emax representa la contractilidad por construcción. Aunque no es el objetivo de esta revisión, se puede también construir curvas de elastancia para diferentes momentos de la diástole. La curva inferior en línea punteada en la Figura 2 corresponde a la relación presión-volumen durante el llene ventricular, o lusitropismo, dependiente de la relajación y distensibilidad de la pared ventricular. $\mathrm{Su}$ forma se explica por una resistencia creciente a la distensión de las diferentes estructuras de su 
pared, produciendo el incremento de la rigidez $(\mathrm{dP} / \mathrm{dV})$ de cámara ${ }^{11}$.

En la Figura 2 se muestra, en línea de trazos y puntos, Emax después de la administración de isoproterenol. En presencia de isoproterenol Emax aumenta (mayor pendiente) y se alcanza antes que Emax control, 180 vs 220 ms $^{10}$. Es decir, Emax se desplaza hacia arriba y a la izquierda, con mayor desarrollo de presión al final de sístole para cada volumen ventricular, así decimos que aumentó la contractilidad. Lo inverso ocurre con una maniobra inótropa negativa como la administración de un bloqueador $\beta$-adrenérgico. Además del cambio de Emax, el cambio de contractilidad también se puede evidenciar por un desplazamiento en paralelo de la curva de Emax en la abscisa con un cambio de $\mathrm{V}_{0}$; un desplazamiento hacia la izquierda de $\mathrm{V}_{0}$ denota un aumento de la contractilidad, mientras que un desplazamiento hacia la derecha, una disminución. Deformaciones geométricas importantes de la cámara ventricular pueden inducir diferentes relaciones entre Emax y $\mathrm{V}_{0}$ que hacen más difícil su interpretación ${ }^{11}$.

De acuerdo a lo anterior, durante un ciclo cardiaco la presión ventricular es función del tiempo transcurrido y del volumen ventricular alcanzado en dicho tiempo. Así, durante la contracción isovolúmica (b-c en línea de trazos, Figura 3), la presión va subiendo desde un punto en una determinada curva de elastancia, según el volumen desde el cual se inicia la contracción (b en Figura 3), a un punto en una curva de elastan-

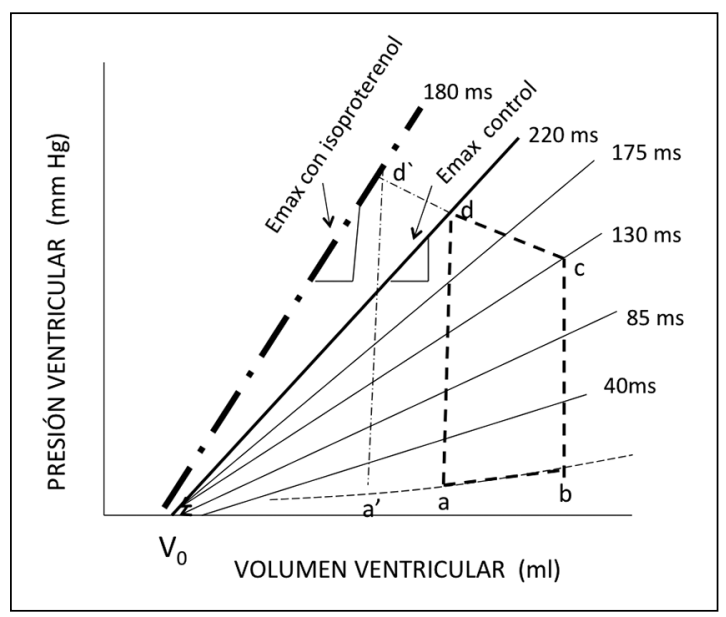

Figura 3. cia superior (cambio de estado contráctil) según el tiempo transcurrido, hasta alcanzar la presión isovolúmica máxima al final de la contracción isovolúmica en una determinada curva de elastancia (c en Figura 3). Igualmente, durante la eyección la presión ventricular va cambiando de una curva de elastancia a otra a medida que el volumen ventricular disminuye (c-d en Figura 3), hasta alcanzar la curva Emax en fin de sístole (d en Figura 3). Luego, durante la diástole la presión desciende pasando de una curva de elastancia a otra inferior hasta alcanzar la máxima relajación y el comienzo del llene ventricular (a en Figura 3). Se construye así un bucle de la relación presión-volumen (bucle a-b-c-d Figura 3 ) el cual queda limitado, por arriba, por la curva de Emax y, por abajo, por la curva de llene ventricular. Al aumentar la contractilidad, el desplazamiento de la curva Emax explica el aumento del volumen expulsivo (c- $d^{\prime}$ en lugar de c-d en Figura 3), obteniéndose el bucle a'-b-c-d'. Debido a que la construcción de curvas isócronas de elastancia requiere una laboriosa manipulación experimental, en la práctica sólo se construye la curva de Emax uniendo con una recta la presión de fin de sístole $\left(\mathrm{P}_{\mathrm{fs}}\right)$ de dos o más bucles obtenidos por cambios de precarga (obstrucción parcial de la vena cava inferior) o postcarga (obstrucción parcial de la raíz aórtica) (Figura 4). La curva de la relación $\mathrm{P}-\mathrm{V}$ de fin de sístole así obtenida tiene una elastancia denominada elastancia de fin de sístole $\left(\mathrm{E}_{\mathrm{fs}}\right)$ y se expresa

$$
\mathrm{E}_{\mathrm{fs}}=\mathrm{P}_{\mathrm{fs}_{\mathrm{s}}} /\left(\mathrm{V}_{\mathrm{fs}}-\mathrm{V}_{0}\right)
$$

en que $\mathrm{P}_{\mathrm{fs}}=$ presión de fin de sístole, $\mathrm{V}_{\mathrm{fs}}=$ volumen de fin de sístole y $\mathrm{V}_{0}=$ intersección de la curva en la coordenada de volumen. $\mathrm{E}_{\mathrm{fs}}$ representa muy aproximadamente a Emax, aunque no idéntica pues no se construyó como isócronas ${ }^{12,13}$, y es la curva habitualmente utilizada en estudios experimentales y clínicos.

La Figura 5 resume las variables hemodinámicas representadas en el bucle P-V ventricular: "a" es la presión al comienzo de la diástole, "b" es la presión al final de la diástole y se usa como subrogante de la precarga, el trazo "a-b" es la evolución de la presión ventricular durante el llene ventricular y define el lusitropismo ventricular ya descrito, " $c$ " es la presión al comienzo de la eyección y se usa como subrogante de la postcarga, el trazo "b-c" corresponde a la contracción isovo- 


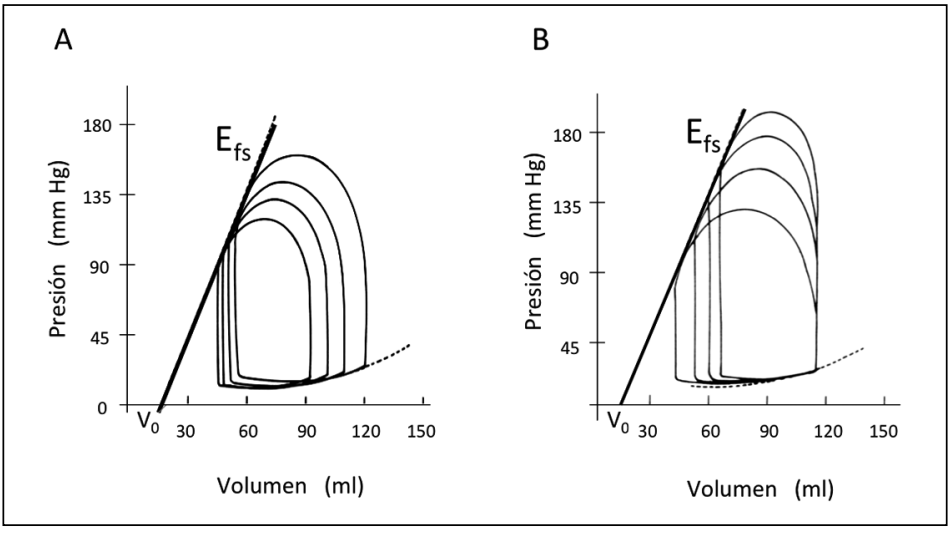

Figura 4.

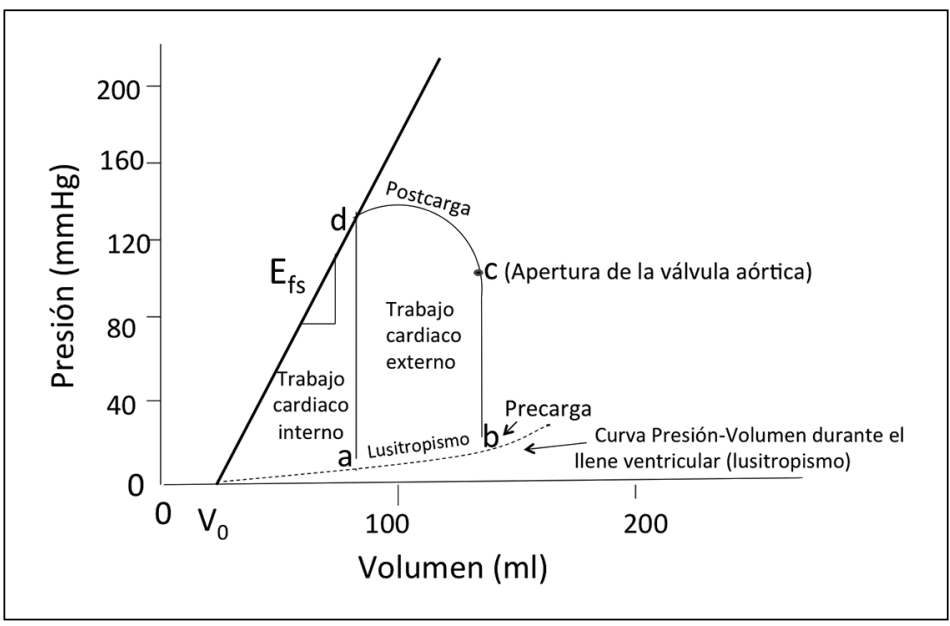

Figura 5.

lúmica, " $\mathrm{d}$ " es la presión de fin de sístole, $\mathrm{P}_{\mathrm{fs}}$, que por definición no puede sobrepasar la curva de Emax. El trazo "d-a" corresponde a la relajación isovolúmica. La proyección de la distancia "b-a" en la abscisa corresponde a la diferencia entre volumen de fin de diástole y volumen de fin de sístole, es decir, el volumen expulsivo, el cual dividido por el volumen de fin de diástole corresponde a la fracción de eyección. El área comprendida en el interior del bucle corresponde al producto de la presión desarrollada durante la expulsión por el volumen expulsivo, llamada trabajo expulsivo, o trabajo externo ventricular. El área a la izquierda del bucle corresponde al trabajo interno del ventrículo (para vencer la elasticidad y viscosidad de los puentes actino-miosina y de otras estructuras del miocardio, especialmente durante la contracción isovolúmica). De esta forma, el bucle P-V ventricular permite mostrar en un solo diagrama la hemodinamia ventricular con sus múltiples componentes.

\section{Medición de cambios de la contractilidad en el hombre. Elastancia y bucle P-V ventricular no invasivo}

El bucle P-V ventricular es el método más eficiente para estimar la contractilidad en el animal de experimentación pero requiere de maniobras invasivas para la medición de la presión ventricular y para modificar precarga y/o postcarga y construir la curva de $\mathrm{E}_{\mathrm{fs}}$ (Figura 4). Recientemente se han desarrollado técnicas para construir el bucle en forma no invasiva en el ser humano y a partir de un solo ciclo cardiaco. La construcción se obtiene (Figura 6) con la medición de los volúmenes de fin de sístole, $\mathrm{V}_{\mathrm{fs}}$, y de fin de diástole, $\mathrm{V}_{\mathrm{fd}}$, mediante 


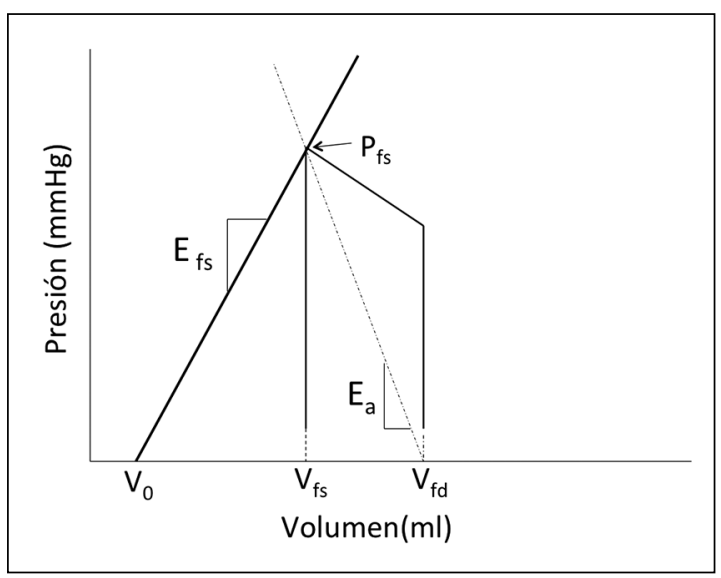

Figura 6.

ecocardiografía, y con la estimación de la presión ventricular de fin de sístole, $\mathrm{P}_{\mathrm{fs}}\left(\mathrm{P}_{\mathrm{fs}}=0,90 \mathrm{x}\right.$ presión arterial sistólica, en ausencia de estenosis aórtica), y de comienzo de la eyección, $\mathrm{P}_{\mathrm{d}}$, a partir de la medición de la presión arterial sistólica y diastólica braquial con esfigmomanómetro. Con estos datos y un algoritmo desarrollado experimentalmente $y$ validado en el ser humano ${ }^{14}$ se obtienen $E_{\mathrm{fs}}$, y $V_{0}$. La Figura 6 muestra un bucle ventricular basal, obtenido de un solo ciclo cardiaco en un individuo normal, semejante al descrito en la Figura 5 pero simplificado. Se observa la curva Presión-Volumen de fin de sístole que une $\mathrm{P}_{\mathrm{fs}}$ con $\mathrm{V}_{0}$. Su pendiente es $\mathrm{E}_{\mathrm{fs}}$, índice de la contractilidad ventricular. En la figura no se observa la curva de llene ventricular debido a la dificultad de obtener la presión ventricular diastólica en forma no invasiva, aunque algunos autores utilizan una estimación no invasiva mediante la ecuación $\mathrm{PFD}=\alpha \operatorname{VFD}^{\beta}(\alpha=$ constante de ajuste de la curva, $\beta=$ constante de rigidez diastólica $)^{15}$. Además, se puede agregar al bucle la curva de elastancia arterial efectiva, $\mathrm{E}_{\mathrm{a}}$ (línea de trazos gruesos en Figura 6) que corresponde a la pendiente negativa del cambio máximo de presión en la aorta (igual a la presión máxima alcanzada en el ventrículo, $\mathrm{P}_{\mathrm{fs}}$, en ausencia de estenosis aórtica) durante la eyección $\left(\mathrm{V}_{\mathrm{fd}}-\mathrm{V}_{\mathrm{fs}}\right)$, y se expresa

$$
\mathrm{E}_{\mathrm{a}}=\mathrm{P}_{\mathrm{fs}} /\left(\mathrm{V}_{\mathrm{fd}}-\mathrm{V}_{\mathrm{fs}}\right)
$$

E es un índice de postcarga (dificultad a la expulsión), determinada principalmente por la resistencia periférica. La razón entre la elastancia arterial efectiva y la elastancia ventricular, $\mathrm{E}_{\mathrm{a}} / \mathrm{E}_{\mathrm{fs}}$, se constituye en un índice del acoplamiento ventrículo-arterial.

Un problema no resuelto es la falta de linealidad de la curva $\mathrm{E}_{\mathrm{fs}}$ con cambios extremos de contractilidad o remodelación ventricular ${ }^{16}$. Dado que $\mathrm{E}_{\mathrm{fs}}$ se calcula a partir de la presión de fin de sístole de un complejo ventricular único, el valor obtenido representa la pendiente a nivel de ese valor pero no necesariamente en el resto de la curva, y su extrapolación a la abscisa para obtener $\mathrm{V}_{0}$ tampoco resulta confiable, lo que dificulta la comparación entre dos situaciones clínicas. Como solución subrogante se mide el volumen ventricular de fin de sístole en la curva $\mathrm{E}_{\mathrm{fs}}$ para una presión ventricular dentro del rango de trabajo clínico, ej $100 \mathrm{mmHg}\left(\mathrm{V}_{100}\right)$, siendo

$$
\mathrm{V}_{100}=100 / \mathrm{E}_{\mathrm{fs}}+\mathrm{Vo}_{\mathrm{O}}
$$

así, a mayor valor de $\mathrm{V}_{100}$ (mayor volumen ventricular de fin de sístole, menor volumen expulsivo para una determinada presión de fin de sístole) menor es la contractilidad, y viceversa ${ }^{16}$.

\section{Ejemplos clínicos}

En la Figura 7 se compara un bucle P-V normal con un bucle como se observa en la insuficiencia cardiaca $^{16,17}$. Nótese que este último está desplazado hacia la derecha en la coordenada de volumen con aumento de $\mathrm{V}_{\mathrm{fd}}$, lo que denota dilatación (remodelación) ventricular. El bucle es más angosto por el menor volumen expulsivo, explicado por: a) menor contractilidad, evidenciada por el desplazamiento de la curva $\mathrm{P}_{\mathrm{fs}}$ hacia la derecha (desplazamiento de $\mathrm{V}_{0}$ ) y por la disminución de $\mathrm{E}_{\mathrm{fs}}$ (menor pendiente) $\mathrm{y}, \mathrm{b}$ ) aumento de $\mathrm{E}_{\mathrm{a}}$, lo que denota aumento de la postcarga probablemente por aumento de la resistencia periférica. En consecuencia, la razón $\mathrm{E}_{\mathrm{a}} / \mathrm{E}_{\mathrm{fs}}$ resulta aumentada, lo que indica desacoplamiento ventrículo-arterial. Nótese que la disminución del volumen expulsivo en la insuficiencia cardiaca no sólo es función de la disminución de la contractilidad. Esta forma gráfica de expresar la función ventricular que incluye contractilidad $\left(\mathrm{E}_{\mathrm{fs}}\right)$, precarga y remodelación $\left(\mathrm{V}_{\mathrm{fd}}\right)$, postcarga $\left(\mathrm{E}_{\mathrm{a}}\right)$, volumen expulsivo $\left(\mathrm{V}_{\mathrm{fd}}-\mathrm{V}_{\mathrm{fs}}\right)$ $\mathrm{y}$ acoplamiento ventrículo-arterial $\left(\mathrm{E}_{\mathrm{a}} / \mathrm{E}_{\mathrm{fs}}\right)$ es de gran utilidad para evaluar el estado clínico del paciente cardiópata y se está introduciendo en el estudio pronóstico de la insuficiencia cardiaca ${ }^{18-23}$. 


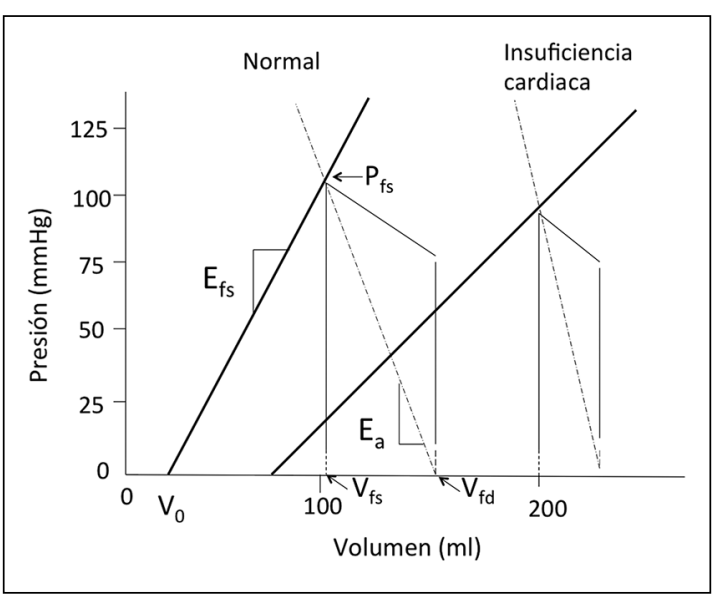

Figura 7.

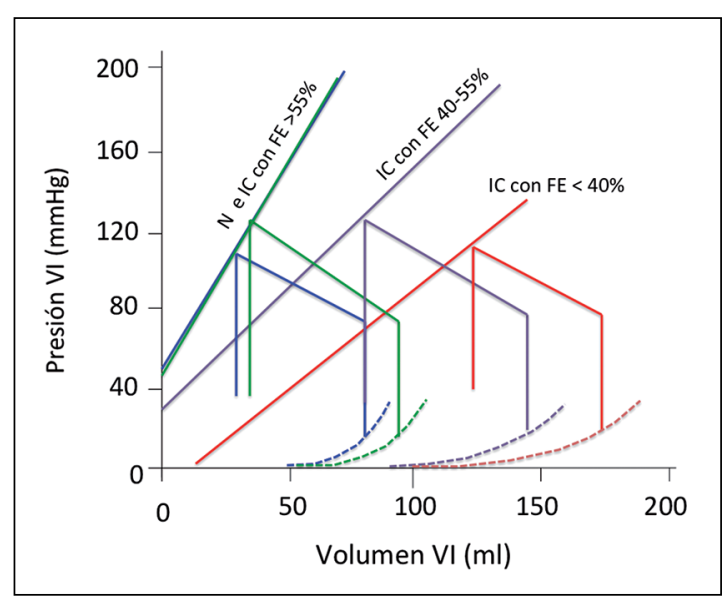

Figura 8.

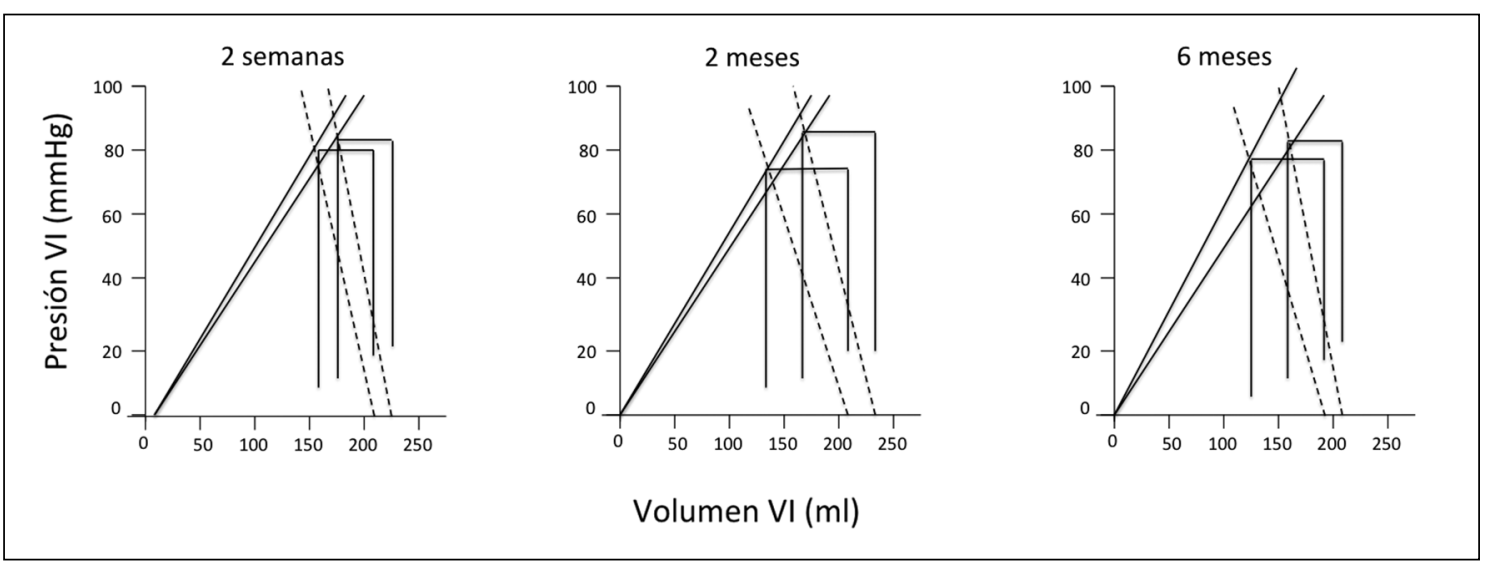

Figura 9.

En la Figura 8 se observan los bucles P-V ventricular en pacientes con diferente fracción de eyección ${ }^{18}$. Nótese la disminución de la contractilidad $\left(\mathrm{E}_{\mathrm{fs}}\right)$ en la insuficiencia cardiaca con preservación de la fracción de eyección (grupo con FE 40-55\%).

En la Figura 9 se observa con este método el efecto beneficioso de carvedilol en pacientes con insuficiencia cardiaca ${ }^{22}$, manifestado por aumento de la contractilidad, disminución de la elastancia arterial y por ende mejoría del acoplamiento ventrículo- arterial, con aumento del volumen expulsivo.

Más recientemente este método demostró la preservación de la contractilidad ventricular a pesar del aumento de la postcarga al disminuir la regurgitación mitral mediante la corrección percutánea de la insuficiencia mitral con el método Mitra Clip $p^{23}$.

Es probable que muchas correcciones y perfeccionamientos a esta metodología ocurran en un futuro próximo, incluso su traducción en la medición de contractilidad regional del miocardio $^{24}$ con técnicas ecocardiográficas o resonancia nuclear magnética para medir cambios de elongación o acortamiento de la pared ventricular ${ }^{25,26}$ y nuevas técnicas para medir la presión ventricular. Tal avance permitirá definir 
en forma precoz y no invasiva el estado contráctil intrínseco del miocardio y desenmascararlo del efecto de la carga ventricular, lo que a su vez permitirá indicar soluciones terapéuticas más tempranas y eficaces.

En todo caso, hoy es más factible resistir la ironía del alumno: "La pregunta no es justa porque ustedes tampoco saben la respuesta", cuando se le preguntó "¿qué es contractilidad?"

\section{Referencias}

1. Katz AM. Physiology of the Heart. Four edition Lippincott Williams \& Wilkins, Philadelphia 2006.

2. Feneley M. Contractility: still searching after all these years. JACC Cardiovasc Imaging 2013; 6: 429-31.

3. Weidemann F, Jamal F, Sutherland GR, Claus P, Kowalski M, Hatle L, et al. Myocardial function defined by strain rate and strain during alterations in inotropic states and heart rate. Am J Physiol Heart Circ Physiol 2002; 283: H792-H9.

4. Ferferieva V, Van den Bergh A, Claus P, Jasaityte R, Veulemans $P$, Pellens $M$, et al. The relative value of strain and strain rate for defining intrinsic myocardial function. Am J Physiol Heart Circ Physiol 2012; 302: H188-H95.

5. Herrmann S, Bijnens B, Störk S, Niemann $\mathrm{M}, \mathrm{Hu} \mathrm{K}$, Liu D, et al. Using simple imaging markers to predict prognosis in patients with aortic valve stenosis and unacceptable highrisk for operation. Am J Cardiol 2013; 112: 1819-27.

6. Shah AM, Claggett B, Sweitzer NK, Shah SJ Anand IS, Liu L, et al. Prognostic importance of impaired systolic function in heart failure with preserved ejection fraction and the impact of spironolactone. Circulation 2015; 132: 402-14.

7. Mentz RJ, Khouri MG. Longitudinal strain in heart failure with preserved ejection fraction: Is there a role for prognostication? Circulation 2015; 132: 368-70.

8. Suga H. Time course of left ventricular pressure-volume relationship under various extents of aortic occlusion. Jpn Heart J 1970; 11: 373-8.

9. Suga H. Time course of left ventricular pressure-volume relationship under various end diastolic volume. Jpn Heart J 1969; 10: 509-15.

10. Suga H, Sagawa K. Instantaneous Pressure-Volume relations hips and their ratio in the excised, supported canine left Ventricle. Circ Res 1974; 35: 117-26.

11. Burkhoff D, Mirsky I, Suga H. Assessment of systolic and diastolic ventricular properties via pressure-volume analysis: a guide for clinical, translational, and basic researchers. Am J Physiol Heart Circ Physiol 2005; 289: H501-H12.

12. Sagawa K, Maughan WL, Suga H, Sunagawa K. Cardiac Contraction and the Pressure-Volume Relationship. Oxford Univ. Press 1988.

13. Kass DA, Maughan WL. From 'Emax' to pressure-volume relations: a broader view. Circulation 1988; 77: 1203-12.

14. Chen $\mathrm{CH}$, Fetics B, Nevo F, Rochitte CE, Chiou KR, Ding PA, et al. Non invasive single-beat determination of left ventricular end-systolic elastance in humans. J Am Coll Cardiol 2001; 38: 2028-34.

15. Klotz S, Hay I, Dickstein ML, Wang J, Maurer MS, Kass DA, et al. Single-beat estimation of end-diastolic pressure-volume relationship: a novel method with potential for noninvasive application. AM J Physiol Heart Circ Physiol 2006; 291: H403-H12.

16. Burkhoff D. Pressure-volume loops in clinical research: a contemporary view. J Am Coll Cardiol 2013; 62: 11736.

17. Ky B, French B, May Khan AM, Plappert T, Wang A, Chirinos JA, et al. Ventricular-arterial coupling, remodeling, and prognosis in chronic heart failure. J Am Coll Cardiol 2013; 62: 1165-72.

18. He KL, Burkhoff D, Leng WX, Liang ZR, Fan L, Wang $\mathrm{J}$, et al. Comparison of ventricular structure and function in Chinese patients with heart failure and ejection fractions $>55 \%$ versus $40 \%$ to $55 \%$ versus $<40 \%$. Am J Cardiol 2009; 103: 845-51.

19. Borlaug BA, Paulus WJ. Heart Failure with preserved ejection fraction: pathophysiology, diagnosis, and treatment. Eur Heart J 2011; 32: 670-9.

20. Lam CSP, Roger VL, Rodeheffer RJ, Bursi F, Borlaug BA, Ommen SR, et al. Cardiac structure and ventricular-vascular function in persons with heart failure and preserved ejection fraction from Olmsted County, Minnesota. Circulation 2007; 115: 1982-90.

21. Bhuiyan T, Helmke S, Patel AR, Ruberg FL, Packman J, Cheung K, et al. Pressure-volume relationships in patient with transthyretin (ATTR) cardiac amyloidosis secondary to V122I mutations and wild-type transthyretin. Transthyretin cardiac amyloid study (TRACS). Circ Heart Fail 2011; 4: 121-8.

22. Maurer MS, Sackner-Bernstein JD, El-Khoury Rumbarger L, Yushak M, King DL, Burkhoff D. Mechanisms underlying improvements in ejection fraction with carvedilol in heart failure. Circ Heart Fail 2009; 2: 189-96.

23. Gaemperli O, Biaggi P, Gugelmann R, Osranek M, Schreuder JJ, Bühler I, et al. Real-time left ventricular 
pressure-volume loops during percutaneous mitral valve repair with the Mitra Clip system. Circulation 2013; 127: 1018-27.

24. Jasaityte R, Claus P, Teske AJ, Herbots L, Verheyden B, Jurcut R, et al. The slope of the segmental stretch-strain relationship as a noninvasive index of LV Inotropy. JACC Cardiovasc Imaging 2013; 6: 419-28.

25. Thorstensen A, Amundsen BH, Dalen H, Hala P, Kiss
G, Aase SA, et al. Strain rate imaging combined with wall motion analysis gives incremental value in direct quantification of myocardial infarct size. EurHeart J Cardiovasc Imaging 2012; 13: 914-21.

26. Bijnens BH, Cikes M, Claus P, Sutherland GR. Velocity and deformation imaging for the assessment of myocardial dysfunction. Eur J Echocardiogr 2009; 10: 216-26. 